\title{
Products Extension Adaptive Design Based on Case Reuse
}

\author{
Ti-chun Wang ${ }^{1,2,3, *}$, Ai-jun Yang ${ }^{1}$ and Shi-sheng Zhong ${ }^{4}$ \\ ${ }^{1}$ Department, University College of Mechanical and Electrical Engineering, Nanjing \\ University of Aeronautics and Astronautics, Nanjing 210016, China \\ ${ }^{2}$ Jiangsu Key Laboratory of Precision and Micro-Manufacturing Technology, \\ Nanjing 210016, China \\ ${ }^{3}$ Department, University Wuxi TJInnova Auto-design Co.,Ltd., Wuxi 214072, China \\ ${ }^{4}$ School of Mechantronics Engineering, Harbin Institute of Technology, Harbin \\ 150001, China \\ wangtichun2010@nuaa.edu.cn

\section{Abstract}

In view of the limit of mechanical products adaptive design in aspects of case representation, retrieval and modification, the representation and reuse technique of knowledge are studied. There are different importance' of matter-element in different design stage, so a kind of similarity calculation method which based on the extension of weighted matter-element is put forward. The product design not only have functional requirements but also structural requirements, so the solving method about incompatible problems and opposite problems in extenics is introduced, a model of products extension adaptive design which based on case reuse is established.

Keywords: Case reuse, Extenics, Adaptive desygn, Scheme design, Matter-element model

\section{Introduction}

Adaptive design which based on basically unchanged of original products, is to change some local features and structures of products, to adapt to some certain requirements. So there are a lot of design experience in original product of case, which can be applied to the design of new products. Adaptive design of mechanical products have been studied by one after another scholars in recent years. Zhao Yanwei [1-2] have proposed an adaptive design method based on/extension transformation. Yan-chao Yin [3],YiFei Chen [4] and Ainong Li [5] have put forward a product adaptive design based on extension theory through dispelling the conflict that appeared in the process of design. Those research mainly focus on contradictoy problems which appear in the process of product adaptive design, there are few research for the reuse of existing case. For choosing the best products that meeting the design requirements from case base is the key of adaptive design, a good case can greatly shorten the circle of product design and reduce the cost of design. Case-based reasoning (CBR) is a widely used method in product scheme design [6]. At the same time, extenics as an emerging intelligent design discipline is laws and methods using formal tool to study contradictions from the qualitative and quantitative perspective, and provides a new method to deal with the optimization of complex product design scheme [7-8]. Dongjun Liu [9], Yong-xiu He [10] and Jianzhou Gong [11] have studied the extension methods based on the matter-element. The problems of extension data mining based on the extension theory are discussed and some methods and models are put forward [12-13]. At present, there are some scholars use extenics 
to study the issues of product design decision and design analysis, and obtain the corresponding results. Such as, JIA Chun-rong [14] have given the extension fuzzy optimization and evaluation models based on the extension theory. This paper put the method of case reuse into the process of adaptive design, and establish a model of product adaptive design based on the extension theory.

\section{Retrieval of Case in Extension Adaptive Design}

\subsection{Establish the matter-element model of product case}

The case matter-element model [7-9] which base on the concept of matter-element in extenics, through the matter-element description of case, is a formal model that unify the quality and quantity together. Due to the functional and structural requirements of design, the case matter-element model of products can be described from the aspects of function and structure, which is case structure matter-element $R_{f}$ and case function matte-element $R_{\mathrm{s}}$, there is a mapping relationship between the two matter-elements

In order to distinguish different kind of cases, $\mathrm{N}$ is used toindicate the name of different case, so the index of function matter-element and structure matter-element are established respectively. Take the structure matter-element as an example, $O$ is an object, $c$ is a characteristic, and $v$ is the value of $O$ about $c$,so $R=(O, c, v)$ is called one-dimensional matter-element as the description of basic element; If the Poject contains multiple characteristics, The array $R$ which contains by $n$ characterstics of object $O$ and the value $v_{i}(i=1,2, \cdots, n)$ of $c_{i}$ corresponded is called $n$-dimensional matter-element.

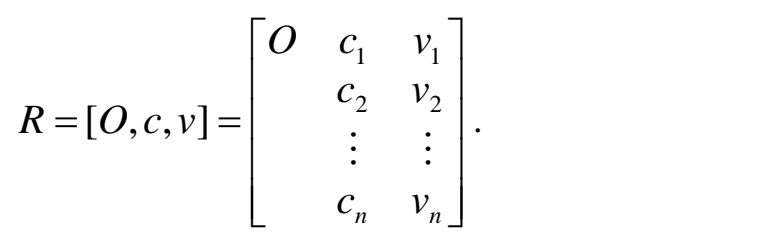

There into, $O$ represents the name of case structure matter-element; $\mathrm{C}$ is the characteristic of $O$ corresponded and $y_{i}$ is the nagnitude of the characteristic $c_{i}$ corresponded, however, the characteristic value of function matter-element generally is a kind of descriptive language. $O, c$ and $v$ is referred as the three elements of matter-element $R . M=(c, v)$ is referred as the characteristic unit. According to $O$, the index of case structure can be established. Thus, by using the matter-element model, the knowledge of product case in adaptive design can be described in qualitative and quantitative way, which makes it easier for the computer representation, storage and processing. At the same time, the extension and extension tansformation of matter-element can be used to solving contradiction problems in adaptive product design.

Design goal is a set of constraints, which reflects the design requirements of products in the engineering semantics, the design requirements, respectively, are established to the function demand matter-element $R_{o f}$ and the structure demand matter-element $R_{\mathrm{os}}$ from the aspects of function and structure. Taking the structure matter-element as an example, then its matter-element model is as fellow: 


$$
R_{o}=\left[O_{o}, c_{o}, v_{o}\right]=\left[\begin{array}{ccc}
O_{o} & c_{o 1} & \left\langle v_{o 1 l}, v_{o 1 m}, w_{o 1}\right\rangle \\
& c_{o 2} & \left\langle v_{o 2 l}, v_{o 2 m}, w_{o 2}\right\rangle \\
& \vdots & \vdots \\
& c_{o n} & \left\langle v_{o n l}, v_{o n m}, w_{o n}\right\rangle
\end{array}\right]
$$

There into, $O_{o}$ represents the name of structure demand matter-element, $c_{o}$ is the characteristic of $O_{o}$ corresponded and $v_{o}$ is the triple of value $c_{o}$ about $O_{o}$, which is indicated as $v_{o i}=\left\langle v_{o i l}, v_{\text {oim }}, w_{\text {oi }}\right\rangle(i=1,2,3 \ldots, n) . v_{\text {oil }}$ and $v_{\text {oim }}$ represents the interval of design requirement of characteristic $c_{o i}$. $w_{o i}$ represents the weight of characteristic $c_{o i}$ (There into, weight represents the importance of attribute in design, the greater the weight, the more important the attribute, and $w_{o 1}+w_{o 2}+\cdots+w_{o n}=1$ ).

\subsection{Similar case retrieval based on extension distance}

The similar case retrieval is the core of case reuse Case model is constituted by characteristics and parameters of product case. By calculating the distance between the case and the design goal, the most similar case based on common characteristics can be selected.

According to the functional and structural requirements, or incomplete of the two aspects, so put the function case matter-element and the structure case matter-element, respectively, similar match with the function demand matter-element and the structure demand matterelement. The degree of correlation among the case matter-elements are based on the similarity of characteristic value of case matter-efenent and the number of similar characteristics. The importance of characteristics are different in different design stages, so according to their importance, some certain weight were given to them. If the design requirement is not exist, its weight is 0 .The previous similan case retrieval only pay attention to the retrieval of value, not put the number of similar eharacteristics of cases into account. There may be difference between the characteristic number of ease matter-element and the demand matter-element, assuming that the namber of their common characteristics is $\mathrm{k}$, then the number of different characteristics between case matte-glement and demand matter-element is $\Delta C_{j}$ :

$$
\Delta C_{j}=\Delta C_{j s}+\Delta C_{j m}=m+n_{j}-2 k_{j}(j=1,2) .
$$

In the formula $\Delta C_{j}=n_{j}-k_{j}, \Delta C_{j s}$ is the number of different characteristics between the $j$ case matter-element and the common matter-element.

In the formata $\Delta C_{j m}=m_{j}-k_{j}, \Delta C_{j m}$ is the number of different characteristics between the $j$ demand matter-element and the common matter-element.

The coefficient of reuse characteristic of case matter-element is $K_{c j}$ :

$$
K_{c j}=1-\left(\Delta C_{j s}+\Delta C_{j m}\right) /\left(m_{j}+n_{j}-k_{j}\right)(j=1,2) .
$$

$K_{c 1}$ and $K_{c 2}$ respectively indicate the coefficient of reuse characteristics of the function matter-element and the structure matter-element. There into, $0 \leq K_{c j} \leq 1$.If the characteristics of case matter-element are similar with the characteristics of demand matter-element exactly, then the coefficient of reuse characteristic of matter-element is 1 ; If there is no common characteristic between the case matter-element and the demand matter-element, then the coefficient of reuse characteristic of matter-element is 0 . 
Taking the case structure matter-element as an example, to calculate the similarity between the case matter-element and the demand matter-element, there are a variety of methods to calculate the similarity between the case structure matter-element and the demand structure matter-element, such as HaiMing distance, OuShi distance, MingKeFu distance and etc. Because the value of characteristic of case generally is the kind of value that meet a certain requirement, for example $v=a$, the design requirement generally is a range, for example $v_{o}=\langle c, d\rangle$, so the distance between point and range is 0 in classical distance, so the calculation method that based on classical distance can't distinguish the difference of cases within the design requirement. In order to describe the difference between the things within class, introducing the extension distance in case reuse to calculate the similarity. The similarity that using extension distance calculated is more accurate than the classical distance acquired, which can distinguish the difference of the similarity of different elements within the same range. The formula of distance between point and interval is

$$
\rho\left(v, v_{o}\right)=|a-(c+d) / 2|-(d-c) / 2
$$

When $a$ at the outside of range $v_{o}=\langle c, d\rangle, \rho\left(v, v_{o}\right)$ is a positive value. When $a$ at the endpoint of range $v_{o}=\langle c, d\rangle$, the value of $\rho\left(v, v_{o}\right)$ 1s zero. When $a$ in the range of $v_{o}=\langle c, d\rangle, \rho\left(v, v_{o}\right)$ is a negative value. When $a$ at the midpoint of range $v_{o}=\langle c, d\rangle, \rho\left(v, v_{o}\right)$ achieve the minimum. Whenthe optimålpoint at the midpoint of range $v_{o}=\langle c, d\rangle$, the similarity degree of characteristic is

$$
S_{2 i}\left(v, v_{o}\right)=((d-c)-|2 a-c-d|) k\left(d Z_{c}\right)(i=1,2,3 \ldots k)
$$

When $S_{2 i}\left(v, v_{o}\right)<0$, there is no simlarity between the characteristic of demand matterelement and the characteristic of ease matter-element. When $S_{2 i}\left(v, v_{o}\right)=0$, the characteristic of demand matter-element and the characteristic of case matter-element are similar to critical. When $S_{2 i}\left(v, v_{o}\right) \in[0,1]$ the characteristic of demand matter-element is similar to the characteristic of case matter-element. The greater the value of $S_{2}\left(v, v_{o}\right)$, the more similar the common characteristic. Only when $S_{2}\left(v, v_{o}\right)=1, a$ exactly is the midpoint of the range $\langle c, d\rangle$.

According to the different 9 mportance of $k$ common characteristics in structure demand, different weights were assigned. The weighted similarity between the case structure matterelement and the corresponding demand structure matter-element is

$$
S_{2}=\sum_{i=1}^{k} m_{i} \delta_{2 i}\left(v, v_{o}\right) .
$$

There into $m_{2}+m_{2}+\cdots+m_{k}=1, k$ is the coefficient of after-weighted reuse characteristic of case matter-element. According to the different importance of case function matter-element and case structure matter-element, the global weighted similarity between case matterelement and the corresponding demand matter-element is

$$
K=\sum_{i=1}^{3} w_{i} K_{c i} S_{i} .
$$

Finally, similar case is selected according to the size of $K$.If the global similarity $K=1$, then the corresponding case is fully comply with the design requirement, which can be applied into new product design directly. Usually the global 
similarity $K$ is the kind of number that greater than zero but less than one, so choose the largest similarity of case, and put it into adaptive modification.

\section{Extension Adaptive Modification of the Similar Case}

Similar case as the reference of problem case, often need to be modified adaptively. Adaptive modification of similar case is a complex process, different kind of product of case often need different kinds of design knowledge and modification method. Previous case modification usually based on rule or constraint reasoning [8], which was largely bounded by professional knowledge and not easy to be achieved in computer. Different kind of modification rules need to be formulated in allusion to different kind of problems, so the method of describing the process of case modification is insufficiency at present. Similar case is adaptively modified in this paper in order to satisfying function and structure requirement

\subsection{Meet the function demand of extension adaptive modification}

It use the $\Delta C_{1 m}$ different characteristics between the demand function matter-element and the common function matter-element and its magnitude to establish the goal/matter-element that named by $G$. It use the structure matter-element of similar case to establish the condition matter-element that named by $L$. Then the incompatible problem of machinery product is established, record it as $P=(G, L)$.At this point, the method of calculating the incompatible problem in extenics has been introduced. There are three ideas to solve incompatible problem: First, the goal remains the same, by transforming the condition to make the incompatible problem to be solved; Second, the condition remain unchanged, through transforming the goal to solve the incompatible problem; Third, by changing the goal and condition to solve the incompatible problem. In order to meet the functional requirements, the goal should be remains the same as far as possible, so we choose the first method in this paper.

Assuming that $c_{o}$ is a evaluation characteristie, $c_{o s}$ is the characteristic that $c_{o}$ needed when the goal $G$ is achieved, $X$ s a positive domain, $X$ is a value domain, and $X_{o} \subset X, C_{o t}$ is the characteristic that the structure matter-element $R$ about $c_{o}$ provided in condition $L$, its value is $C_{o t}\left(R_{o}\right)$, then $P_{o} \varepsilon_{o} * l_{o}$ is called the nuclear problem of $P$, there into, $g_{o}=\left(R_{o}, c_{o s}, X_{o}\right), l_{o} \neq\left(R_{o}, c_{o t}, c_{o t}\left(R_{o}\right)\right)$, do $W=\left\{l \mid l=\left(R, c_{o}, c_{o}(R)\right)=\left(R, c_{o}, x\right)\right\}$, then $X_{o}\left(X_{o} \subset X\right)$ is a positive domain, The compatible function $k(x)$ of $l$ about $c_{o}$ is established. The extension set is 1

$$
\tilde{E}(T)=\left\{\left(x, y, y^{\prime}\right) \mid l \in T_{w} W, y=K(l)=k(x) \in(-\infty,+\infty), y^{\prime}=T_{k} K\left(T_{l} l\right) \in(-\infty,+\infty)\right\}
$$

$K_{o}(P)=K\left(l_{o}\right)=k\left[c_{o t}\left(R_{o}\right)\right]$ is called consistency degree of problem $P$.If $K_{o}(P)<0$, then in the extension sets $\tilde{E}(T), T=\left(T_{w}, T_{k}, T_{l}\right)$, there into $T_{w}$ is the transformation of domain $W, T_{k}$ is the transformation of compatible function, and $T_{l}$ is the transformation of element $l$. If $P$ is an incompatible problem, and there is an extensible transformation $T=\left(T_{w}, T_{k}, T_{g}\right)$ make $T_{K} K\left(T_{l_{o}} l_{o}\right)=K^{\prime}\left(T_{l_{o}} l_{o}\right)=K^{\prime}\left(l_{o}^{\prime}\right)>0$, then $T$ is the solutional transformation of incompatible problem $P$. 


\subsection{Meet the structure demand of extension adaptive modification}

If the existing condition $L_{1}$ can't meet the question of $G_{1}$ and $G_{2}$ at the same time, then establish the opposite problem $P_{1}=\left(G_{1} \wedge G_{2}\right) * L_{1}$, there into, $G_{1}$ is composed by the demand different structure matter-element, $G_{2}$ is composed by the common structure matter-element and $L_{1}$ is composed by the existing interface. At this point, the extension model of opposite problem has been built.

Assuming that $c_{1}$ is a evaluation of characteristic, $R_{1}$ and $R_{2}$ are the object that the goal of $G_{1}$ and $G_{2}$ involved, $c_{1 s}$ is the characteristic needed by $R_{1}$ and $R_{2}$ about $c_{1}, X_{10}$ and $X_{20}$ represent positive domain, $X_{1}$ and $X_{2}$ represent value domain, there into $X_{10} \subset X_{1}, X_{20} \subset X_{2}$, the object unit is $g_{10}=\left(R_{10}, c_{1 s}, X_{10}\right)$ and $g_{20}=\left(R_{20}, c_{1 s}, X_{20}\right)$ and the condition unit is $l_{10}=\left(R_{10}, c_{o t}, c_{o t}\left(R_{10}\right)\right)$ and $l_{20}=\left(R_{20}, c_{o t}, c_{o t}\left(R_{20}\right)\right)$ then the nuclear problem of $P_{1}$ is $P_{10}=\left(g_{10} \wedge g_{20}\right) *\left(l_{10} \wedge l_{20}\right)$.Make a dual extension set

$$
\begin{aligned}
& \tilde{E}\left(l_{1}, l_{2}\right)=\left\{\left(\left(l_{1}, l_{2}\right), y, y^{\prime} \mid l_{1} \in T_{U} U, l_{2} \in T_{v} V,\left(l_{1}, l_{\mathcal{L}}\right) \in T_{U} U \times T_{V} V\right.\right. \\
& y=K\left(l_{1}, l_{2}\right)=k\left(x_{1}, x_{2}\right), y^{\prime}=T_{K} K\left(T_{l_{1}} l_{1}, T_{q_{2}} l_{2} k^{\prime}\left(x_{1}^{\prime}, x_{2}^{\prime}\right)\right\}
\end{aligned}
$$

There into, $U=\left\{l_{1} \mid l_{1}=\left(R_{1}, c_{1}, x_{1}\right)\right\}, \quad V=\left\{l_{2} P_{2}=\left(R_{2}, c_{1}, l_{2}\right)\right\} . K_{o}\left(P_{1}\right)=k\left[c_{o t}\left(R_{10}\right), c_{o t}\left(R_{20}\right)\right]$ is called the coexistence degree of problem $P_{1}$.If $K_{o}\left(P_{1}\right)>0$, then $P_{1}$ is called the coexistence problem, if $K_{o}\left(P_{1}\right)<0$, then $P_{1}$ is called the opposite problem, if $K_{o}\left(P_{1}\right)=0$, then $P_{1}$ is called the critical problem.

If $K_{o}\left(P_{1}\right)<0$, and there is an extensible transformation $T=\left(T_{l_{1}}, T_{l_{2}}, T_{k},\left(T_{U}, T_{V}\right)\right)$ make $T_{K} K\left(T_{l_{1}} l_{1}, T_{l_{2}} l_{2}\right)=K^{\prime}\left(T_{1} l_{1}, T_{l_{2}} l_{2}\right)=k^{\prime}\left(x_{1}^{\prime}, x_{2}^{\prime}\right)>0$, then $T$ is the solution transformation of opposite problem $P_{1}$.

\subsection{The process of products extension adaptive design based on case reuse}

The process of products extension adaptive design which based on case reuse mainly contain the retrieval of similar case and the adaptive modification of similar case. First, the existing product case and the design goal are established to matter-element model from the aspects of function and structure respectively, and the weight is distributed according to its importance. Then the case base is retrieved according to the similarity of case and goal, the similarity between the goal matter-element and the case matter-element is calculated quantitatively. Finally, select the largest similarity of case and put it into adaptative design. The process is shown in Figure 1. 


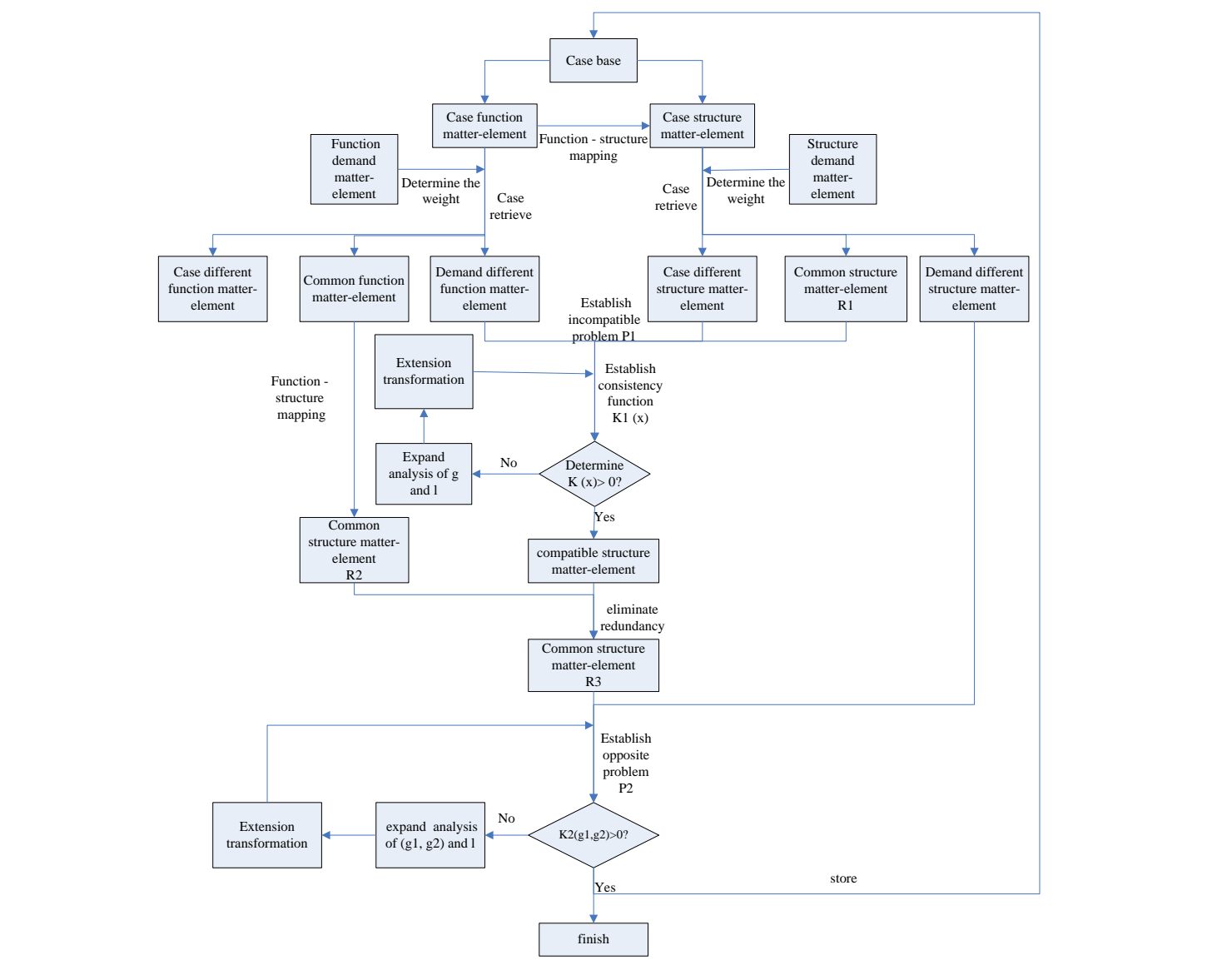

Figure 1. The process of products adaptive design based on the case reuse

The steps of products adaptive design based on case reuse are described as follows:

Step1 Acquiring the function and structure needs of product design process, by extracting the demand characteristigs and its mapping, the function demand matter-element model and the structure demand matter-element model are established respectively and its weights are distributed. According to the aspects of function and structure, the case base is established to matter-element model respectively.

Step2 According to the proposed similarity calculation model to calculate the similarity between case matter-element and demand matter-element, and select the largest global similarity of case matter-element. According to the number of different characteristics between case matter-element and demand matter-element, and whether $S_{1 i}\left(v, v_{o}\right)$ and $S_{2 i}\left(v, v_{o}\right)$ are satisfy the similarity threshold respectively or not, the case and design requirements are divided into case function matter-element, common function matter-element and demand different function matter-element from the angle of function; the case and the design requirements are divided into case structure matter-element, common structure matterelement and demand different structure matter-element from the angle of structure.

Step3 In order to meet the function requirements, to establish the extension model of incompatible problem $P=(G, L)$.There into, the goal function matter-element of design requirements that named by $G$ is consisted by the demand different function matter-element, 
the existing condition structure matter-element that named by $L$ is consisted by the selected global similarity of case structure matter-element.

Step4 Establishing the compatibility function $K(x)$, which is the decision function of compatibility problem between condition and goal. If $K(x)>0$, then $P$ is a compatibility problem, and the selected largest global similarity of case matter-element is the compatible structure matter-element, directly to step 7. If $K(x)<0$, then in step 5.

Step5 Give expand analysis to $G$ and $L$, and give extensible transformation to the expanded matter-element which based on the divergent, enlarge shrinks, contain and conjugate characteristic of matter-element.

Step6 Determine whether $K(x)>0$ or not again, if $K(x)<0$, then in step 5, if $K(x)>0$, then in step 7.

Step7 Give analysis to the common structure matter-element $R_{2}$ and the compatible structure matter-element, to remove its structure redundancy.

Step8 In order to meet the structure requirement\$, to establish the opposite problem $P=\left(G_{1} \wedge G_{2}\right)$, there into, the problem structure matter-element that named by $G_{1}$ is consisted by the demand different structure matter-element, the problem structure matter-element that named by $G_{2}$ is consisted by common structurematter-élement.

Step9 According to the analysis of conditions and denand, to establish the coexistence function $K(x)$. Determine whether $K(x) \& 0$ or not, in $K(x)>0$, then $P$ is a coexistence problem, directly to the end. If $K(x)<0$, then in step 10 .

Step10 Give expand analys 8 to $G_{1} 、 G_{2}$ and $L_{1}$, and give extensible transformation to the expand matter-element. ${ }^{\bullet}$

Step11 Determine, whether $K(x)>\rho$ or not again, if $K(x)<0$, then in step10, if $K(x)>0$, then difectly to the end.

\section{Instance Application}

Taking the type selection of a hydraulic turbine for example, give further explanation to extension adaptiye design method that based on case reuse. According to the design requirement and the corresponding weight of function characteristics, to establish the functional demand matter-element that named by $R_{f o}$.

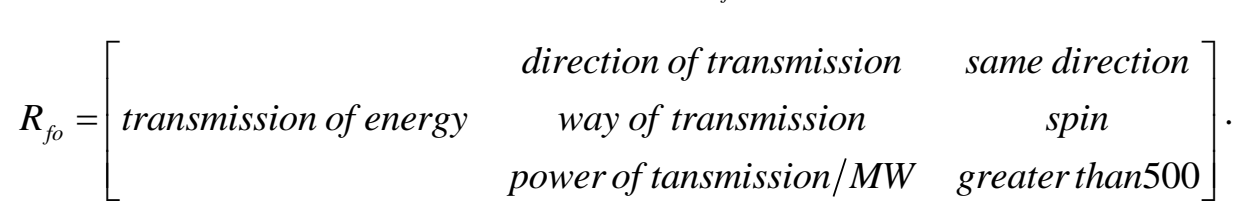

By retrieving the case base, the case that meet the constraint condition is acquired, its function matter-element model is

$$
R_{f}=\left[\begin{array}{ccc} 
& \text { direction of transmission } & \text { same direction } \\
\text { transmission of energy } & \text { way of transmission } & \text { spin } \\
& \text { power of tansmission } / M W & 300
\end{array}\right] .
$$


According to the function - structure mapping, the structure matter-element that the function matter-element $R_{f}$ corresponded is

$$
R_{s}=\left[\begin{array}{cc}
\text { material } & \text { forge18MnMoNb } \\
\text { spindle diameter } / \mathrm{mm} & \varphi 1880 \\
\text { flange diameter } / \mathrm{mm} & \varphi 2400 \\
\text { flange height } / \mathrm{mm} & 550 \\
\text { lang } / \mathrm{mm} & 7909 \\
\text { center hole } / \mathrm{mm} & \varphi 1600
\end{array}\right] .
$$

Due to the not satisfied characteristics and the value of function matter-element, then the demand different function matter-element that named by $R_{f d o}$ is established, there into, $R_{f d o}=$ [transmission of energy transmission of power $/ M W$ greaterthan500].

According to the condition that the spindle can't meet the requirements of transmission power, the incompatible problem that named by $P=(G, L)$ is established. There into, the goal matter-element that named by $G$ is consisted by $R_{f d o}$, the condition structure matter-element that named by $L$ is consisted by $R_{s}$. because the size of the transmitted power is influenced by the material, diameter and length of spindle, so by changing to high intensity of spindle material appropriately increasing the diameter and shortening the length of spindle, the resistance shear stress of the spindle can be increased. The condition matter-element that after extension transformed is

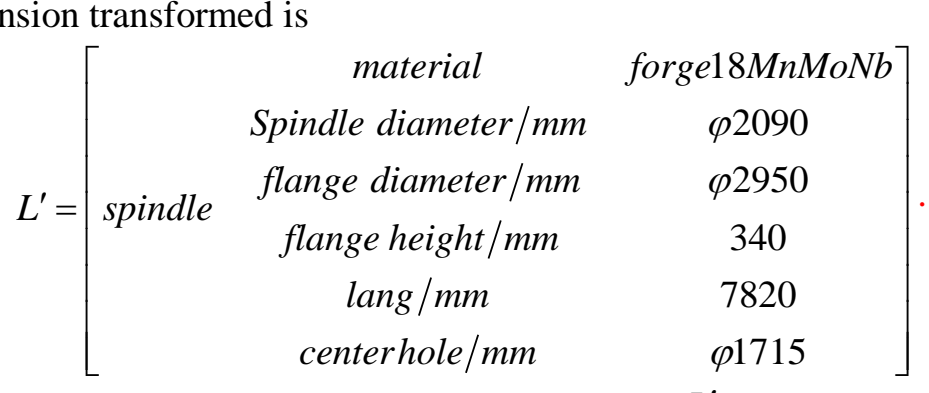

The structure strength calcutation shows that $L^{\prime}$ meet the demand of goal matter-element, so $L^{\prime}$ becomes to the compatible structure matter-element. In the same way according to the requirement of wheel structure the demand structure matter-element is established. The fully met structure matter-element that named by $R_{s}$ is acquired through the formula (6).

$$
R_{s}=\left[\begin{array}{cc}
\text { specified size } / \mathrm{mm} & 8000 \\
\text { max outside diameter } / \mathrm{mm} & 8620 \\
\text { min lumen diameter } / \mathrm{mm} & 2010 \\
\text { weight of upper canopy } / \mathrm{mm} & 106.8 \\
\text { weight of band } / \mathrm{mm} & 34.866
\end{array}\right] \text {. }
$$

Because of the change of spindle diameter, the size of other parts that connected with spindle also need to be changed. For example, the structure of wheel also need to be changed, so the opposite problem appearing. The opposite problem named by $P=\left(G_{1} \wedge G_{2}\right) * L$ is established, there into, the goal matter-element named by $G_{1}$ is consisted by the compatible structure matter-element, the goal matter-element named by $G_{2}$ is consisted by the similar 
structure matter-element, and the condition matter-element named by $L$ is consisted by the existing interface condition. If the diameter of spindle stays the same, then $G_{1}$ and $G_{2}$ will be coexistence problem through the analysis of goal matter-element $G_{1}$ and $G_{2}$. By changing the material and length of spindle, the functional requirements can be satisfied. Then the goal matter-element that after extensional transformed is

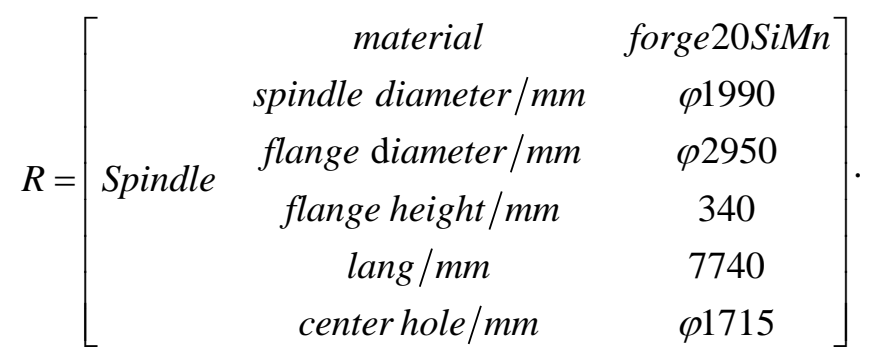

So the spindle that satisfying the requirement of function and structure is

$$
R=\left[\begin{array}{cc}
\text { material } & \text { forge20SiMn } \\
\text { spindle diameter } / \mathrm{mm} & \varphi 1990 \\
\text { flange diameter } / \mathrm{mm} \\
\text { flange height } / \mathrm{mm} \\
\text { lang } / \mathrm{mm} \\
\text { center hole } / \mathrm{mm}
\end{array}\right.
$$

This article which based on the matter-element theory, established the formal matterelement model for the knowledge of product adaptive design, the problem and case in case reuse were described in the combination of quantitative and qualitative way. According to the different design stages and different importance of design requirements, different weights were assigned, the weighted modets of case reuse which based on extension distance were proposed, and the accyracy of case retrieval was improved. By introducing the solving method of incompatible problem and opposite problem in extenics from the angle of case reuse, the extension adaptive design model of mechanical product was established, and the feasibility of this process is verified by application case.

\section{Acknowledgements}

This research was supported by the National Natural Science Foundation Youth Fund of China (No. 51005114); The Fundamental Research Funds for the Central Universities, China (No. NS2012047); The Researeh Fund for the Doctoral Program of Higher Education, China (No. 20112302130003); A Project Funded by Priority Academic Program Development of Jiangsu Higher Education Institutions (PAPD); China Postdoctoral Science Foundation (No. 2013M540445).

\section{References}

[1] Y. W. Zhao and G. X. Zhang, "A New Integrated Design Method Based On Fuzzy Matter-Element Optimization", Journal of Materials Processing Technology, vol. 129, no. 1-3, (2012), pp. 612-618.

[2] N. Su, Y. Zhao, L. Xing, et al., "A variant module division method on Extension logic for assembly process", 2008 ASME International Mechanical Engineering Congress and Exposition, Boston, Massachusetts, USA, (2008) October 31-November 6, pp. 1-10. 
[3] Y. -c. Yin, L. -f. Sun and C. Guo, "A policy of conflict negotiation based on fuzzy matter element particle swarm optimization in distributed collaborative creative design", Computer-Aided Design, vol. 40, no. 10-11, (2008), pp. 1009-1014.

[4] Y. Chen, D. Liu, J. Wu, et al., "Extension based clustering method an approach to support adaptable design of the product", Proceedings of the 2007 International Manufacturing Science And Engineering Conference, Atlanta, Georgia, USA, (2007) October 15-17, pp. 1-9.

[5] A. Li, J. Jiang, J. Bian, et al., "Combining the matter element model with the associated function of probability transformation for multi-source remote sensing data classification in mountainous regions", ISPRS Journal of Photogrammetry and Remote Sensing, vol. 67, (2012), pp. 80-92.

[6] W. Tichun, C. Bingfa and B. Liangfeng, "Extension Configuration Model of Product Scheme Design Based on Axiomatic Design”, China Mechanical Engineering, vol. 23, no. 19, (2012), pp. 2269-2275.

[7] J. Chun-rong and Z. Jun, "Based on Fuzzy Weight Matter Element to Evaluate the Water Quality of Jialing River in Nanchong, China", Procedia Environmental Sciences, vol. 11, (2011), pp. 631-636.

[8] L. Yang, "Assessment of City Environmental Quality in Western China Based on Matter Element Extensiona Case Study Of Chongqing", Energy Procedia, vol. 5, (2011), pp. 619-623.

[9] D. Liu and Z. Zou, "Water quality evaluation based on improved fuzzy matter-element method", Journal of Environmental Sciences, vol. 24, no. 7, (2012), pp. 1210-1216.

[10] Y. -x. He, A. -y. Dai, J. Zhu, et al., "Risk assessment of urban network planning in china based on the matterelement model and extension analysis", International Journal of Electrical Rower\& Energy Systems, vol. 33, no. 3, (2011), pp. 775-782.

[11] J. Gong, Y. Liu and W. Chen, "Land suitability evaluation for development using a matter element model: A case study in Zengcheng, Guangzhou, China", Land Use Policy, vol. 29, no. 2, (2012), pp. 464-472.

[12] T. Wang, S. Zhao and B. Chen, "Association Rule Extension Mining and Reuise in Scheme Design of Largescale Hydraulic Turbines”, Information: An International Interdisciplinary Journal, vol. 15, no. 6, (2012), pp. 2403-2409.

[13] M. -H. Wang, Y. -F. Tseng, H. -C. Chen, et al., "A novel custering algorithm based on the extension theory and genetic algorithm", Expert Systems with Applications, vol. 36, no 4, (2009), pp. 8269-8276.

[14] J. Chun-rong and Z. Jun, "Evaluation of Regional Circular Econony)Based on Matter Element Analysis", Procedia Environmental Sciences, vol. 11, (2011), pp. 637-642.

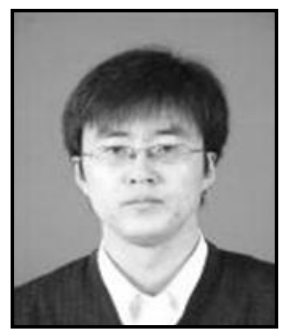

Tiehự Wang. He received his M.Sc. in mechanical engineering (2005) and PhD in mechanical engineering (2009) from University. Now he is associate professor of School of Mechantronics Engineering, Nanjing Uniersity of Aeronautics and Astronautics. His current research interests include different aspects of Artificial Intelligence, Knowledge Engineering and Extension Engineering.

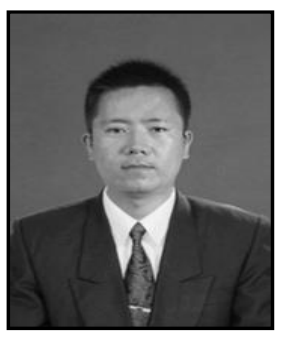

Aijun Yang. He received his B.Sc. in mechanical engineering (2011) from University. Now he is a graduate student of School of Mechantronics Engineering, Nanjing University of Aeronautics and Astronautics. His current research interests include different aspects of Knowledge Engineering and Extension Engineering. 


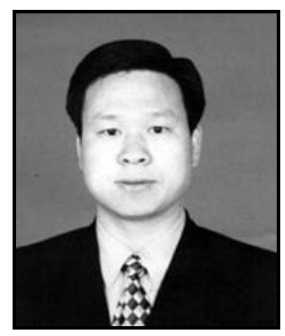

Shisheng Zhong. He received his M.Sc. in mechanical engineering (1992) and PhD in mechanical engineering (1995) from University. Now he is full professor of School of Mechantronics Engineering, Harbin Institute of Technology. Since 2007 he is vice principal of University . His current research interests include different aspects of Artificial Intelligence,Knowledge Engineering and Complex Equipment Condition Monitoring and Repair Decision.

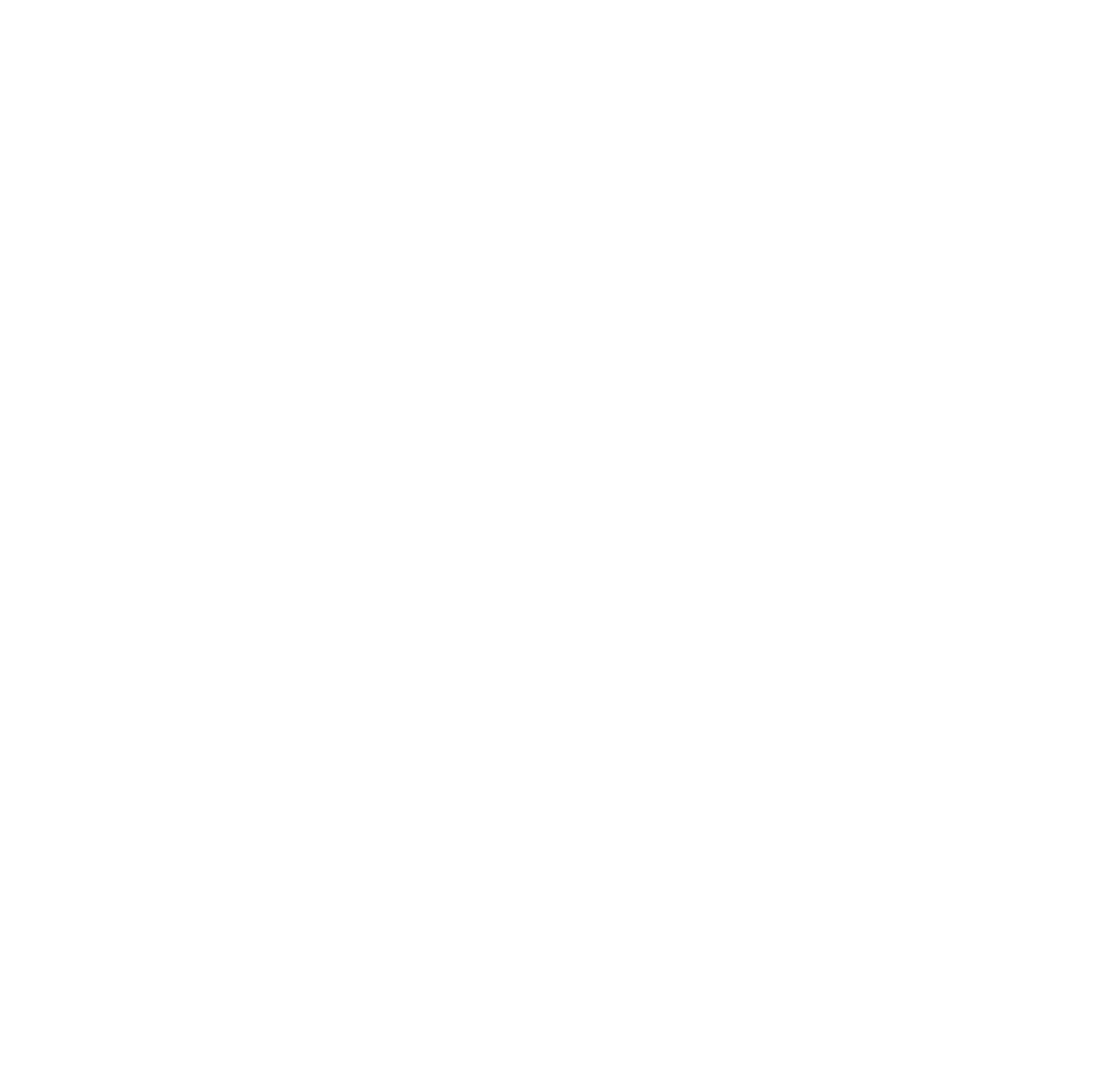

